\title{
Intergalactic medium
}

\author{
Raghunathan Srianand \\ IUCAA, Post Bag 4, Ganeshkhind, Pune 411007, India \\ email:anand@iucaa.ernet.in
}

\begin{abstract}
The absorption lines seen in the spectra of high redshift QSOs are important tools for studying the early evolution of galaxies and intergalactic medium. In this presentation I briefly review various available observations, our understanding of different types of absorption systems, and highlight some of the issues that can be addressed with ELTs.
\end{abstract}

Keywords. quasar:absorption lines, Galaxies: Intergalactic medium, Galaxies: high-redshift

\section{Introduction}

The absorption lines seen in the spectra of high redshift quasars are produced by the gas associated with galaxies as well as by the gas located in the desolate regions of space (called intergalactic medium) where no star can be found. The study of these absorption lines is one of the most sensitive and powerful tools to understand the early evolution of galaxies and the intergalactic medium (IGM). In the past decade or so QSO absorption lines were believed to be the only means by which one could study the high- $z$ protogalaxies. Once $10 \mathrm{~m}$ class telescopes became available and the high- $z$ galaxies were accessible using Lyman-break techniques the role of the absorption systems detected in QSO spectra underwent a major revision. Absorption lines seen in the spectra of QSO are now used to study the physics of low luminosity galaxies, investigate feedback effects (such as metal pollution and entropy injection) of galaxy formation and QSOs and investigate the thermal and ionization history of the universe. In addition, high resolution high signal-to-noise spectra can be used to perform fundamental tests of current theories not only in astrophysics, but also more generally in physics. In particular, it is possible to probe the time evolution of the cosmic microwave background (Srianand et al. 2000) and that of dimensionless fundamental constants (Murphy et al. 2003; Srianand et al. 2004).

Traditionally QSO absorption lines are classified into intrinsic and intervening systems. Intervening absorption systems are believed to be produced by material located by chance somewhere in the universe in between us and the quasar. Any gas component with $\mathrm{H} \mathrm{I}$ column densities in the range $10^{12}-10^{22} \mathrm{~cm}^{-2}$ can be detected in absorption. Based on the $\mathrm{H}$ I column densities alone these systems are further classified into (See Fig. 1):

(i) Lyman- $\alpha$ systems: The most numerous systems have $\log N(\mathrm{H} \mathrm{I}) \leqslant 17.00$. They are highly ionized, have very low density and low metallicities. They are believed to trace the low density intergalactic medium (see top panel of Fig. 1).

(ii) Lyman limit systems (LLS): Rare systems that are optically thick at the $\mathrm{H}$ i ionization potential $\left(912 \AA\right.$ ) and have therefore $\mathrm{H}$ I column densities $N(\mathrm{H} \mathrm{I})>2 \times 10^{17} \mathrm{~cm}^{-2}$ (see lower panel in Fig. 1). They are associated with strong metal absorption lines and are believed to arise in the halos of galaxies.

(iii) Damped Lyman- $\alpha$ systems (DLAs): About $10 \%$ of Lyman limit systems show damping wings in the Lyman- $\alpha$ lines (i.e., $\log (\mathrm{H} \mathrm{I}) \geqslant 20.0)$ (see middle panel in Fig. 1). 

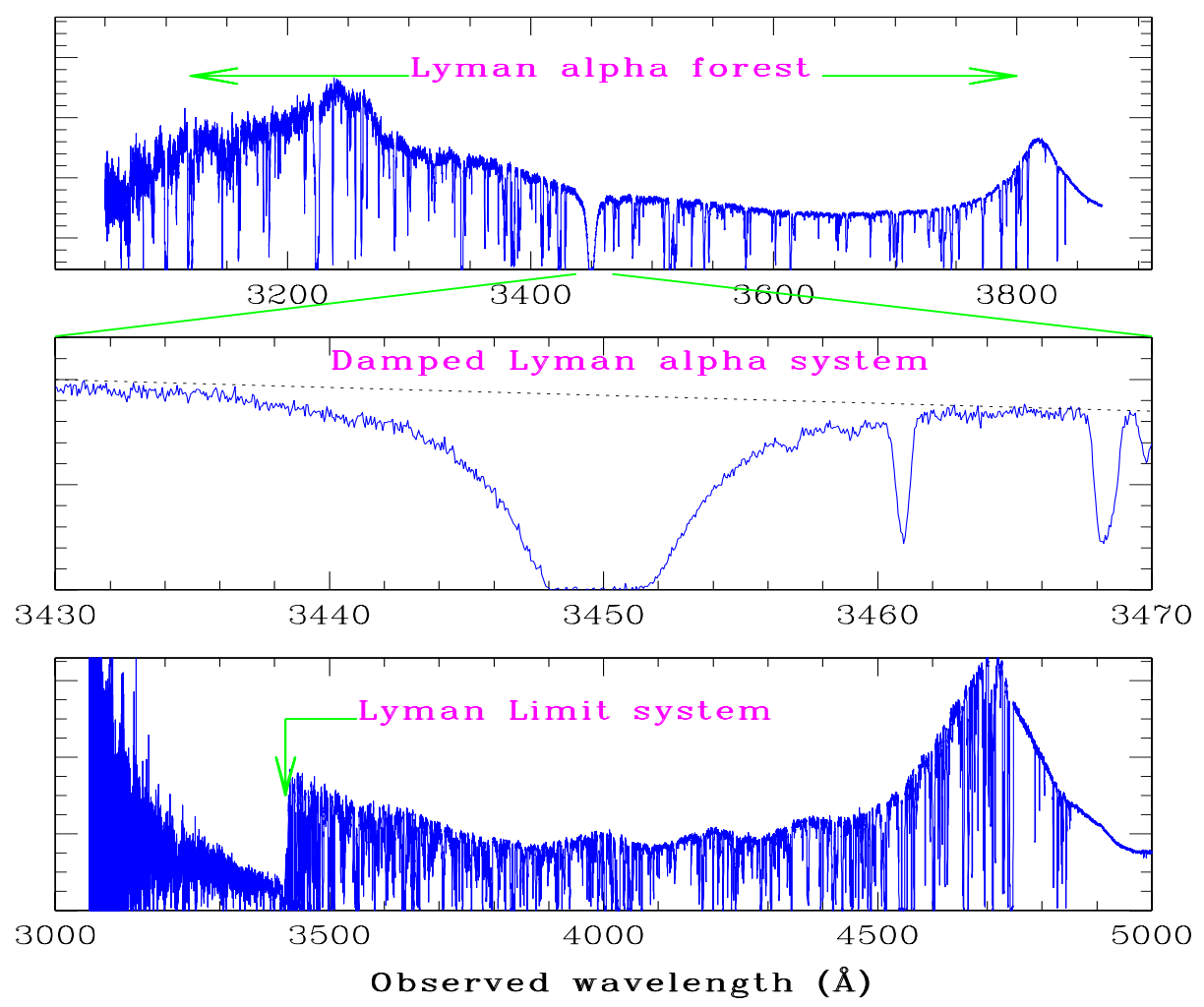

Figure 1. Classification of intervening absorption systems. The top panel shows Lyman- $\alpha$ forest absorption systems seen toward $z_{\mathrm{em}}=2.1$ QSO 1101-260. In the frame work of standard structure formation models these are produced by evolving baryonic density perturbations. A damped Lyman alpha system, that probably traces a very high density peak, is seen at $z_{\text {abs }}=1.83$. A blowup of this is shown in the middle panel. The lower panel shows the spectrum of $z_{\mathrm{em}}=3.8$ QSO HE 2347-4342. A sudden break in the spectrum noticed at $\lambda \simeq 3422$ is caused by the Lyman limit system at $z_{\mathrm{abs}}=2.75$.

Hydrogen is neutral and therefore detailed study of metallicities is possible. These systems are believed to trace proto-galactic disks and therefore are unique probes of galaxy formation history.

Even though there is growing evidence for the presence of heavy elements in the intergalactic medium (and therefore in the Lyman- $\alpha$ systems), the latter two categories of system are usually referred to as metal line systems as they always show absorption lines of a large number of elements in different ionization stages from $\mathrm{C}$ I to $\mathrm{C}$ IV or $\mathrm{O}$ I to $\mathrm{O}$ VI.

In this presentation, I will review the recent understanding of the DLAs and the intergalactic medium and highlight issues that can be addressed with ELTs.

\section{Damped Lyman- $\alpha$ systems (DLAs)}

DLAs are characterized by the presence of damping wings in the Lyman- $\alpha$ absorption line that suggests high $\mathrm{N}(\mathrm{H} \mathrm{I})$ : typically $N(\mathrm{H} \mathrm{I}) \geqslant 10^{20} \mathrm{~cm}^{-2}$. In the local universe, gas with such column densities is usually associated with galaxies. At high redshifts DLAs 
represent a major reservoir of neutral hydrogen (Wolfe 1995) and the presence of heavy elements suggests that DLAs are located in over-dense regions where star formation activity may take place (Pettini et al. 1997). The large $\mathrm{N}(\mathrm{H} \mathrm{I})$ also means that most of the heavy elements will be either in neutral or in singly ionized states. This allows us to investigate the chemical enrichment history of the absorbing gas without worrying too much about ionization corrections.

Metallicity: Early spectroscopic studies of DLAs concentrated mainly on the estimation of average metallicity and its time evolution. The abundance of Fe and its depletion in DLAs are usually estimated from $\mathrm{N}(\mathrm{Zn} \mathrm{II}) /(\mathrm{H} \mathrm{I})$ and $\mathrm{N}(\mathrm{Fe} \mathrm{II}) / \mathrm{N}(\mathrm{Zn}$ II) respectively (Lu et al. 1996a; Pettini et al. 1997; Prochaska \& Wolfe, 2002, Khare et al. 2005; DessaugesZavadsky et al. 2006). The basic idea is that Zn and Fe are co-produced; Zn depletes little and provides the actual abundance, while the relative depletion of Fe with respect to $\mathrm{Zn}$ provides the dust content.

The inferred metallicities typically vary between $\log Z=-2$ and 0 for $2 \leqslant z_{\text {abs }} \leqslant 3$ with a median of $\simeq-1.0$. The measured depletions range between 0 and -1.6 with a median value of -0.5 . If one defines dust content as $\kappa=10^{[\mathrm{Zn} / \mathrm{H}]}\left(1-10^{[\mathrm{Fe} / \mathrm{Zn}]}\right)$ then the median dust content in a typical DLA corresponds to $\kappa=0.07$ (Ledoux et al. 2003). This is less than $10 \%$ of what is seen in the Galactic ISM for similar neutral hydrogen column densities. The mean metallicity shows statistically significant redshift evolution. However, the rate of increase in metallicity is much lower than one expects from the age metallicity relation for Galactic-disk stars (Prochaska et al. 2003; Kulkarni et al. 2005). Clearly the DLAs at low- $z$ seem to sample a chemically slowly evolving population.

Studying the star-formation activities in DLAs is very important for understanding the formation and evolution of galaxies. Recently Wolfe et al. (2003) have shown that, even if DLAs sustain only a moderate star-formation activity, they will contribute appreciably to the global star-formation rate (SFR) at high redshifts. The SFR in DLAs can be estimated either by detecting the galaxies responsible for the DLAs or by inferring the intensity of the UV field in DLAs using the population levels of atomic and molecular species. In the latter case, it is important to have a clear understanding of the physical conditions in the gas to derive an accurate estimate of the SFR. In the case of the Galactic ISM, rotational excitations of $\mathrm{H}_{2}$ and the fine-structure excitations of $\mathrm{C}$ I, C II, O I and Si II are used to derive the physical state of the absorbing gas. Echelle spectroscopy with $10 \mathrm{~m}$ class telescopes allows us to detect some of these transitions in DLAs and perform the analysis as we do in the case of Galactic ISM (Srianand et al. 2005a).

Molecular hydrogen: If the DLAs are associated with star-forming regions, we expect to detect Lyman and Werner band absorption lines of $\mathrm{H}_{2}$. However, $\mathrm{H}_{2}$ is rarely detected in DLAs (see Ge \& Bechtold 1999). A systematic search for molecular hydrogen in DLAs at high redshift $\left(z_{\text {abs }}>1.8\right)$, using UVES at the VLT, down to a detection limit of typically $N\left(\mathrm{H}_{2}\right) \sim 2 \times 10^{14}$, has resulted in detection in $\sim 15 \%$ of the cases (see Petitjean et al. 2000; Ledoux et al. 2003). The detectability of $\mathrm{H}_{2}$ is found to be independent of $\mathrm{N}(\mathrm{H} \mathrm{I})$ and $\mathrm{H}_{2}$ detections are usually amongst those with the highest metallicities and depletion factors. This directly demonstrates that a large amount of dust is present in the components where $\mathrm{H}_{2}$ is detected. There are cases where the depletion is very similar to our Galactic ISM (Petitjean et al. 2002). As the total $\mathrm{N}(\mathrm{H})$ in these components is low, these DLAs do not produce appreciable reddening of the background QSOs. The mean $\mathrm{H}_{2}$ molecular fraction in DLA systems is generally small (typically $\log f<-1$ ) and similar to what is observed in the Magellanic Clouds. Approximately 50 percent of the systems have $\log f<-6$ : this is probably a consequence of a reduced formation of $\mathrm{H}_{2}$ on dust grains (probably because the gas is warm, $T>1000 \mathrm{~K}$ ) and/or of an enhanced ionizing flux relative to that which is observed in our Galaxy. 
Physical state of $\mathrm{H}_{2}$ gas: Usually in cases where $\mathrm{H}_{2}$ absorption is detected $\mathrm{C}$ I, $\mathrm{C} \mathrm{I}^{*}$, $\mathrm{C} \mathrm{I}^{* *}$ and $\mathrm{C} \mathrm{II}^{*}$ absorption lines are also detected. The mean kinetic temperature of the gas corresponding to DLA subcomponents in which an $\mathrm{H}_{2}$ absorption line is detected, derived from the ortho-to-para ratio $(153 \pm 78 \mathrm{~K})$, is higher than that measured in the ISM $(77 \pm 17 \mathrm{~K})$ and the Magellanic Clouds $(82 \pm 21 \mathrm{~K})$. Typical pressure in these components (corresponding to $T=100-300 \mathrm{~K}$ and $n_{\mathrm{H}}=10-200 \mathrm{~cm}^{-3}$ ), measured using the $\mathrm{C}$ I fine-structure excitation, are higher than those measured along ISM sight lines. From the column densities of the high-J rotational levels, we can show that the typical radiation field in the $\mathrm{H}_{2}$ bearing components is of the order of, or slightly higher than, the mean UV field in the Galactic ISM. Determination of the electron density in the gas with $\mathrm{H}_{2}$ and $\mathrm{C}$ I show that the ionization rate is similar to that of a cold neutral medium (CNM) in a moderate radiation field. The excitation of $\mathrm{C}$ II in these systems is consistent with the physical parameters derived from the excitation of $\mathrm{H}_{2}$ and $\mathrm{C}$ I. This, together with the fact that we see $\mathrm{H}_{2}$ in $\sim 15 \%$ of the DLAs, can be used to conclude that DLAs at $z>1.9$ could contribute as much as $50 \%$ of the star formation seen in Lyman break galaxies (LBGs) (Srianand et al. 2005a).

Cooling rate: $\mathrm{C}$ II* absorption is more frequently detected $(\sim 50 \%)$ compared to $\mathrm{C}$ I or $\mathrm{H}_{2}$ (Wolfe et al. 2003; Srianand et al. 2005a). If the gas is a cold neutral medium (CNM) then $\mathrm{C} \mathrm{II}^{*}$ directly gives the cooling rate. The required heating rate in systems with $\mathrm{C} \mathrm{II}^{*}$ absorption lines suggests DLAs can contribute appreciably to the global star-formation rate (Wolfe et al. 2003). However, interpretation of $\mathrm{C} \mathrm{II}^{*}$ absorption is difficult in the absence of independent temperature indicators. The physical conditions could be similar to that in the CNM gas of the Galaxy. However, the absence of $\mathrm{C}$ I absorption lines and the presence of Al III absorption lines with profiles similar to those of singly ionized species, suggest an appreciable contribution from warm (WNM) and/or partially ionized gas (Lizst 2002; Srianand et al. 2005a). The absence of $\mathrm{H}_{2}$, for the level of metallicity and dust depletion seen in these systems, is consistent with low densities (i.e. $\mathrm{n}_{\mathrm{H}} \leqslant 1 \mathrm{~cm}^{-3}$ ) for a radiation field similar to the mean Galactic UV field. The $50 \%$ of the systems that do not show $\mathrm{C}$ II $^{*}$ absorption originate mainly from WNM and/or partially ionized gas (see also Wolfe et al. 2004).

$21 \mathrm{~cm}$ absorption: The nature of and physical conditions in the $\mathrm{H} \mathrm{I}$ gas can also be probed by $21 \mathrm{~cm}$ absorption lines. The search for $21 \mathrm{~cm}$ absorption in DLAs at $z_{\text {abs }} \geqslant 2$ have mostly resulted in null detections with typical spin temperatures $\geqslant 10^{3} \mathrm{~K}$ (Kanekar \& Chengalur 2003). Based on the lack of $21 \mathrm{~cm}$ absorption in most of the systems it is inferred that DLAs actually trace a warmer H I gas. Even in the low- $z$ DLAs where the 21 $\mathrm{cm}$ absorption is detected the inferred spin temperatures are a bit higher than those seen in our Galaxy. However, one of the possible uncertainties could be the covering factor of the $\mathrm{H}$ I gas.

DLA galaxy connections: If DLAs sustain moderate star formation activities then one would expect to detect the DLA galaxies in emission. At low redshifts, the galaxies detected close to the DLAs are mostly sub-L ${ }_{*}$ galaxies (with luminosity ranging between 0.02 to $1.2 \mathrm{~L}_{K}^{*}$ ) (Rao et al. 2003). These DLA galaxies span a mixture of morphological types from patchy, irregular, and low surface brightness to spiral galaxies. At high- $z$, despite may attempts, only a handful of DLAs have been detected in emission until now (Moller \& Warren 1993; Djorgovski et al. 1996; Moller et al. 2004; Christensen et al. 2004; Weatherley et al. 2005). However, most of these systems seems to have high metallicity and may not trace typical DLAs.

Gas kinematics: Kinematics of the low ionization lines that trace the H I gas can be used to infer the nature and evolution of underlying gravitation potential in DLA galaxies. It has also been shown that the profiles of the lines arising in neutral gas show evidence 
for rotation (e.g., Prochaska \& Wolfe 1997). However, hydrodynamical simulations have shown that the high redshift progenitors of present-day galactic disks could look like an aggregate of well separated dense clumps. In fact, the kinematics seen in the absorption line profiles of DLAs could be explained by relative motions of the clumps with little rotation (Haehnelt et al. 1998; Ledoux et al. 1998). Recently a correlation was also found between the velocity widths (W) of low-ionization lines in DLAs and average metallicity (Z) (Ledoux et al. 2005). Within the observational uncertainties it has been shown that while the slope of the correlation is not evolving with $z$ the average $Z$ measured for systems with given $\mathrm{W}$ is higher at lower redshifts. This suggests that galaxy halos of a given mass/velocity tend to become more metal-rich with time. This is consistent with the results of Savaglio et al. (2005) for $0.4 \leqslant z \leqslant 1.0$ galaxies selected from the Gemini Deep Survey.

Science cases for ELTs: Spectroscopic observations using ELTs will allow the detection of Ly $\alpha$ and/or $\mathrm{H} \alpha$ emission from typical DLAs (i.e. those with low metallicity). This will allow us to probe the star formation activities in lower mass halos if the above mentioned velocity-metallicity correlation reflects the underlying mass-metallicity relation. In addition high resolution spectra with ELTs will allow the detection of absorption produced by the excited fine-structure levels of C I, C II, O I and Si II, even from low density regions. At lower densities, depending on $z$, some of these excitations are mainly dominated by the $\mathrm{CMB}$ and one will be able to measure directly the redshift evolution of the background temperature. $\mathrm{H}_{2}$ formation is independent of dust in a low density high temperature gas. Model calculations suggests that $\mathrm{H}_{2}$ should be present at the level $\mathrm{N}\left(\mathrm{H}_{2}\right) \simeq 10^{12} \mathrm{~cm}^{-2}$ in DLAs (Srianand et al. 2005b). Blue sensitive spectrographs, if available on ELTs, will directly detect $\mathrm{H}_{2}$ in most of the DLAs.

\section{Low density IGM}

Physical conditions in the low density IGM at $1.8 \leqslant z \leqslant 6$ are probed using the numerous Ly- $\alpha$ absorption lines seen in the spectra of distant quasars, the so-called Lyman- $\alpha$ forest (Fig. 1). The progress in this field is triggered mainly by the availability of high resolution spectroscopic observations and hydrodynamical simulations. These absorption lines have for a long time been believed to be the signature of discrete and localized intergalactic clouds photoionized by the UV-background. The present day understanding is that most of the Lyman- $\alpha$ lines with column density, $\mathrm{N}(\mathrm{H} \mathrm{I}) \leqslant 10^{14} \mathrm{~cm}^{-2}$, originate in quasi-linear density fluctuations in which the hydrogen gas is in ionization equilibrium with a metagalactic UV-background produced by star-forming galaxies and quasars. Non-linear effects are unimportant and therefore the properties of the Lyman- $\alpha$ forest are described well (for a fixed background cosmology) by just three basic ingredients: quasi-linear theory for the growth of baryonic structure, a UV radiation field, and the temperature of the gas (see for example: Muecket et al. 1996; Bi \& Davidsen 1997; Choudhury et al. 2001a,b). This paradigm is impressively confirmed by full hydrodynamical simulations (Cen et al. 1994; Miralda-Escudé et al. 1996; Hernquist et al. 1996; Theuns et al. 1998). The simple picture of a low density IGM allows us to probe various issues related to galaxy formation.

Temperature and equation of state: In the early 1990s it was general practice to decompose the Lyman- $\alpha$ forest into multiple Voigt profiles (e.g., Carswell et al. 1991). A lower envelop in the distribution of $\mathrm{N}(\mathrm{H} \mathrm{I})$ vs. b (the velocity dispersion) was evident in some cases (e.g., Khare et al. 1997; Lu et al. 1996b). It was realized that for a perturbed low density ionized hydrogen gas in photoionized equilibrium adiabatic expansion will give raise to a temperature density relation (Hui \& Gnedin 1997) that can reveal itself in 
the form of an $\mathrm{N}$ vs b relation. Numerical simulations have confirmed the existence of a lower envelope in the $\mathrm{N}$ vs. b scatter plot. This has been used by various groups to obtain the temperature of the IGM at mean densities (Schaye et al. 1999; Ricotti et al. 2000; Zaldarriaga et al. 2001; McDonald et al. 2001). Different groups make different inferences for the temperature evolution of the IGM; however, what is important to note is that the temperature of the IGM at the mean density is in the range $10^{4} \leqslant T(\mathrm{~K}) \leqslant 3 \times 10^{4}$ for $2 \leqslant z \leqslant 4$. This has been used to argue that hydrogen reionization occurred below $z \simeq 9$ (Theuns et al. 2002). Had the reionization occurred at higher $z$, adiabatic cooling would have brought the temperature much below the measured value at $z=4$. The CMB data from the WMAP satellite (Kogut et al. 2003), however, point to an earlier epoch of reionization in the range $11<z<30$. Thus either new heating sources (other than photo-heating) or multiple episodes of reionization with heating at low $z$ provided by, say, the He II reionization induced by QSOs (Hui \& Haiman, 2003), are needed to explain the IGM temperature at $z \sim 2-4$. There is an indication of an increase in $\mathrm{T}$ around $z \simeq 3.2$, confirming this with more numerous QSO spectra could constrain the He II reionization epoch.

Matter power-spectrum: As the underlying physics of the low density IGM is simple, it can potentially be used to study the mater density power-spectrum (Croft et al. 1998). The observed flux-power spectrum depends on (i) Cosmological parameters (i.e. the linear matter power spectrum and cosmological parameter such as $\mathrm{H}_{0}$ and $\Omega_{m}$ ) and (ii) IGM parameters (such as temperature, radiation field and the effective equation of state of the gas). Thus model independent recovery of the matter power spectrum from the Lyman- $\alpha$ forest will in general be degenerate. However, consistency checks of the spectra simulated under a given cosmological setting can be used to distinguish between different matter power-spectra. Constraints from the Lyman- $\alpha$ forest observations are used with those obtained from other studies such as SDSS and WMAP to get tighter constraints on the index of the primordial power-spectrum, mass of the neutrinos, nature of dark matter and mass of potential warm dark matter candidates (see Seljak et al. 2005).

Metals in the IGM: As Lyman- $\alpha$ absorption originates from the low density regions, the metals need to be transported from the star forming objects. Thus the amount and distribution of metals in the Lyman- $\alpha$ forest provides information about feedback from galaxy formation. The Lyman- $\alpha$ absorption with $\mathrm{N}(\mathrm{H} \mathrm{I}) \geqslant 10^{14.5} \mathrm{~cm}^{-2}$ usually shows detectable C IV absorption (Tytler et al. 1995; Songaila \& Cowie 1996). Deriving metallicity from these observations depends on the ionization corrections and usually $[\mathrm{C} / \mathrm{H}] \sim-2.5$ is derived for $z \simeq 3$. One of the important questions is 'what is the volume filling factor of the metals?' As under-dense space occupies most of the volume, measuring metallicity in the low density regions is very important. For example, if metals are found in the gas filling the voids, then they must have been produced in the very early universe by objects more of less uniformly distributed in space. Given the expected low metalicities, direct detection of metals from such low neutral hydrogen optical depth (i.e. $13 \leqslant \log \mathrm{N}(\mathrm{H} \mathrm{I}) \leqslant 14$ ) is currently impossible and usually metallicity is estimated using pixel statistics (Ellison et al. 2000; Schaye et al. 2003; Aracil et al. 2004). The presence of metals in systems with $\tau(\mathrm{H} \mathrm{I}) \geqslant 1$ is more or less confirmed by different groups. Also there seems to be agreement on the fact that there is no evolution in metallicity until $z \simeq 5$ (Songaila 2005). However, the presence of metals in very low density regions has not yet been convincingly proved.

$U V$ background radiation: The major uncertainty in converting the observed column densities of ions into a metallicity is the unknown ionization corrections. The UVbackground that causes the photo-ionisation is dominated by massive stars and quasars (Haardt \& Madau 1996; Giroux \& Shapiro 1996). The amplitude of the corresponding photo-ionisation rate as a function of redshift, $\Gamma(z)$, and the relative importance of the 
different sources, are relatively uncertain. In simulations, assuming a standard Big Bang baryon fraction, the value of $\Gamma_{12}$ has to be between 0.3 and 2 at a redshift $z=2-3$ in order to reproduce the observed Lyman- $\alpha$ forest properties, such as the mean transmission and the column density distribution (Miralda-Escudé et al. 1996; Rauch et al. 1997; Zhang et al. 1997; Choudhury et al. 2001b). An independent way of estimating $\Gamma$ is the proximity effect. Locally, the UV-field may be dominated by a single source, such as a bright quasar, leading to a deficit of absorption lines sufficiently close to the quasar. Because the amount of absorption is in general increasing with redshift, this reversal of the trend for redshifts close to the emission redshift of the quasar is called the 'inverse' or 'proximity' effect (Carswell et al. 1982; Murdoch et al. 1986). The strength of this effect depends on the ratio of the ionisation rates from the quasar and the UV-background, and since the quasar's ionisation rate can be determined directly, $\Gamma_{12}$ can be inferred. This method was pioneered by Bajtlik, Duncan \& Ostriker (1988) but more recent data have yielded a wide variety of estimates. Scott et al. (2000) collected estimates from the literature which vary over almost an order of magnitude at $z=3$, i.e. $1.5 \leqslant \Gamma_{12} \leqslant 9$. However, it is possible that the density field around QSOs may be different (higher density) from the mean IGM values; in that case the derived ionization rates will be upper limits (Rollinde et al. 2005).

Recent studies of the transverse proximity effect by Croft (2004) and Schirber et al. (2004) also suggest excess absorption over that predicted by models which assume the standard proximity effect and isotropic quasar emission. If this is not due to an increase in density close to the quasar, it might imply that the quasar light is strongly beamed, or alternatively that the quasar is highly variable. Observations of the IGM transmission close to LBGs show that the IGM contains more neutral hydrogen than the global average at comoving scales $1<\mathrm{r}(\mathrm{Mpc})<5 \mathrm{~h}^{-1}$ (Adelberger et al. 2003). As the UV photons from the LBGs cannot alter the ionization state of the gas at such large distances, it is most likely that the excess absorption is caused by the enhanced IGM density around LBGs. It is worth noting that various hydrodynamical simulations have trouble reproducing this so-called galaxy proximity effect.

Science cases for ELTs: Some of the issues that can be addressed with ELTs are: the direct detection of strong metal transitions from $\tau(\mathrm{H} \mathrm{I}) \leqslant 1$ gas and the volume filling factor of metals. Good wavelength coverage is needed to estimate the metallicity, this is because to keep the ionization corrections under control we need multiple transitions. Detecting absorption produced by singly ionized metal lines at $z \geqslant 6$ in the spectrum of QSOs or GRBs will allow us to probe the early reionization and metal pollution in the universe. The other interesting science case will be the investigation of the effect of foreground galaxies in the spectra of background QSOs/bright LBGs (like the studies of Adelberger et al. 2005). This will give a direct handle on feed back from star forming galaxies. Spectroscopy of closely spaced high- $z$ low luminosity QSOs and/or bright LBGs will allow us to map the tomography of Lyman- $\alpha$ forest at smaller angular scales.

\section{Time variation of fundamental constants}

Some of the modern theories of fundamental physics, such as SUSY, GUT and Superstring theory, allow possible space and time variations of the fundamental constants, thus motivating an experimental search for such a variation. QSO absorption lines can be used to probe the time evolution of fundamental constants (Bahcall et al. 1967). Murphy et al. (2003), applying the Many Multiplet method (MM method) to 143 complex metal line systems, claimed a non-zero variation of the fine-structure constant, $\left(\alpha=e^{2} / \hbar c\right)$ : $\langle\Delta \alpha / \alpha\rangle=(-0.57 \pm 0.11) \times 10^{-5}$ for $0.2 \leqslant z \leqslant 3.5$, where $\Delta \alpha / \alpha=\left(\alpha_{z}-\alpha_{0}\right) / \alpha_{0}$, with $\alpha_{0}$ 


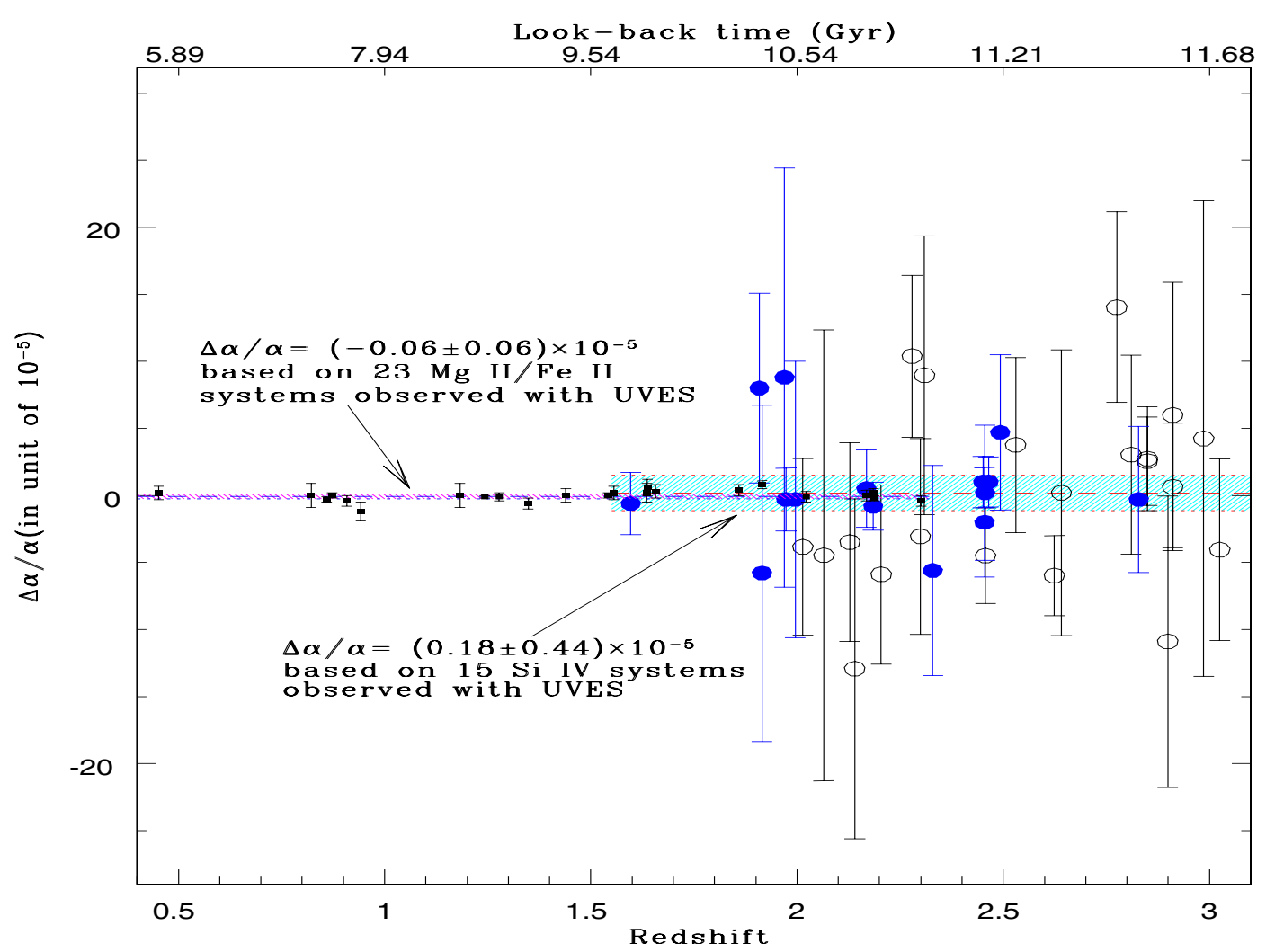

Figure 2. $\Delta \alpha / \alpha$ measurements from the UVES sample: The plot shows the measured values of $\Delta \alpha / \alpha$ versus the absorption redshifts of the systems. The square point show our result from the MM method using the Mg II/Fe II system, and the narrow shaded region represents the $3 \sigma$ allowed range (Srianand et al. 2004; Chand et al. 2004). The filled circles are our measurements using Si IV doublets from UVES (Chand et al. 2005) and open circles are the measurements from KECK/HIRES by Murphy et al. (2001).

being the present value and $\alpha_{z}$ its value at redshift $z$. This result, if true, would have very important implications for our understanding of fundamental physics, and has therefore motivated new activity in the field.

The alkali-doublet method that uses the Si IV doublet is a clean method for constraining the variation in $\alpha$ using spectral lines because it uses transitions from the same species. No variation is detected using this method and the tightest constraint obtained to date is $\Delta \alpha / \alpha=(0.15 \pm 0.44) \times 10^{-5}$ at $z \sim 2$ (Chand et al. 2005). Recent attempts using the MM method (or its modified version) applied to very high quality UVES spectra have also resulted in null detections (Fig. 2). The analysis of Fe II multiplets and Mg II doublets in a homogeneous sample of 23 systems has yielded a stringent constraint, $\Delta \alpha / \alpha=(-0.06 \pm 0.06) \times 10^{-5}$ (Chand et al. 2004; Srianand et al. 2004). Modified MM method analysis of $z_{\mathrm{abs}}=1.15$ toward HE $0515-4414$, that avoids possible complications due to isotopic abundances, has resulted in $\Delta \alpha / \alpha=(0.01 \pm 0.17) \times 10^{-5}$ (Quast et al. 2004). Levshakov et al. (2005) have obtained $\Delta \alpha / \alpha=(0.4 \pm 1.5) \times 10^{-6}$ using modified MM method in two systems.

Clearly all studies based on VLT-UVES data are in contradiction with the conclusions of Murphy et al. (2003). It is most likely that the differences cannot be attributed to 
uncertainties in the UVES wavelength calibration or the differences in various fitting procedure (Chand et al. 2006). Even though studies based on UVES data are consistent with no variation in $\alpha$ they still allow smaller variations in excess of what is found based on the Oklo phenomenon(Fujii et al. 2000). Also one of the uncertainties with the present data will be the non-uniqueness of the Voigt profile decomposition. $R \geqslant 100000$ spectra will be needed for this purpose. However, such spectra can be obtained for only one or two sources with available telescopes (see Chand et al. 2006). Thus it is important to further improve the constraints at higher redshifts using very high resolution $(R \geqslant 100000)$ high signal to noise ratio spectra $(S / N \geqslant 100)$ with very good wavelength calibration accuracy. ELTs are ideally suitable for this purpose. To cover a good redshift range and to be able to use various transitions from a given ion, a wide wavelength coverage will be needed.

\section{Acknowledgements}

I thank the organizers for a very interesting meeting and the IAU for providing travel support.

\section{References}

Adelberger, K.L., Steidel, C.C., Shapley, A.E. \& Pettini, M. 2003, ApJ 584, 45

Adelberger, K.L., Shapley, A.E., Steidel, C.C., Pettini, M., Erb, D.K., Reddy, N.A. 2005, ApJ 629, 636

Aracil, B., Petitjean, P., Pichon, C. \& Bergeron, J. 2004, A\&A 419, 811

Bahcall, J.N., Sargent, W.L.W. \& Schmidt, M. 1967, ApJ 149, L11

Bajtlik, S., Duncan, R. \& Ostriker, J. 1988, ApJ 327, 570

Bi, H. \& Davidsen, A.F. 1997, ApJ 479, 523

Carswell, R.F., Whelan, J., Smith, M., Boksenberg, A. \& Tytler, D. 1982, MNRAS 198, 91

Carswell, R.F., Lanzetta, K.M., Parnell, H.C. \& Webb, J.K. 1991, ApJ 371, 36

Cen, R., Miralda-Escude, J., Ostriker J.P. \& Rauch M. 1994, ApJ 437, L83

Chand, H., Srianand, R., Petitjean, P. et al. 2004, A $₫ A$ 417, 853

Chand, H., Petitjean, P, Srianand, R. \& Aracil, B. 2005, A 3 A 430, 47

Chand, H., Srianand, R., Petitjean, P., Aracil, B., Quast, R. \& Reimers, D. 2006, A\&A in press (astro-ph/0601165)

Choudhury, T., Padmanabhan, T. \& Srianand, R. 2001a, MNRAS 322, 561

Choudhury, T., Srianand, R. \& Padmanabhan, T. 2001b, ApJ 559, 29

Cristensen, L., Sanchez, S.F., Janke, K., et al. 2004, A\&\&A 417, 487

Croft, R. 2004, ApJ 610, 642

Croft, R.A.C., Weinberg, D.H., Katz, N. \& Hernquist, L. 1998, ApJ 495, 44

Dessauges-Zavadsky, M., et al. 2006, A\&\&A 445, 93

Djorgovski, S.G., Pahre, M.A., Bechtold, J. \& Elston, R. 1996, Nature 382, 234

Ellison, S., Songaila, A., Schaye, J. \& Pettini, M. 2000, AJ 120, 1175

Fujii, Y., et al. 2000, Nuc. Phy. B 573, 377

Ge, J., Bechtold, J. 1999, in: C.L. Carilli, S.J.E. Radford, K.M. Menten \& G.I. Langston (eds.), Highly redshifted Radiolines, ASP conf. ser. (San Francisco: ASP) Vol. 156, p. 121

Giroux, M.L. \& Shapiro, P.R. 1996, ApJS 102, 191

Haardt, F. \& Madau, P. 1996, ApJ 461, 20

Hernquist, L., Katz, N., Weinberg, D.H. \& Miralda-Escude, J., 1996, ApJL 457, 51

Hui, L. \& Gnedin, N. 1997, MNRAS 292, 27

Hui L. \& Haiman, Z. 2003, ApJ 596, 9

Kanekar, N. \& Chengalur, J. 2003, A\&A 399, 857

Kogut, A., Spergel, D.N., Barnes, C., et al. 2003, ApJS, 148, 161

Khare, P., Srianand, R., York, D. G. et al. 1997, MNRAS 285, 167

Khare, P., Kulkarni, V.P., Lauroesch, J.T. et al. 2005, ApJ 616, 86

Kulkarni, V.P., Fall, S.M., Lauroesch, J.T. et al. 2005, ApJ 618, 68 
Ledoux, C., Petitjean, P., Bergeron, J., Wampler, E. J. \& Srianand, R. 1998, A\&A 337, 51

Ledoux, C., Petitjean, P. \& Srianand, R. 2003, MNRAS 346, 209

Ledoux, C., Petitjean, P., Moller P., Fynbo J. \& Srianand R. 2005, (astroph/0504402)

Levshakov, S., Centurin, M., Molaro, P \& D’Odorico, S. 2005, A\& $A$ 434, 827

Liszt, H. 2002, A\&A 389, 393

Lu, L., Sargent, W.L.W., Barlow, T., Churchill, C.W. \& Vogt S. 1996a, ApJ 107, 475

Lu, L., Sargent, W.L.W., Womble, D. S. \& Takada-Hidai, M. 1996b, ApJ 472, 509

McDonald, P., Miralda-Escude, J., Rauch, M., Sargent, W.L.W., Barlow, T.A. \& Cen, R. 2001, ApJ 562, 52

Miralda-Escude, J., Cen, R., Ostriker, J. \& Rauch, M. 1996, ApJ 471, 582

Moller, P. \& Warren, S.J. 1993, ApJ 27043

Moller, P., Fynbo, J.P.U. \& Fall, S.M. 2004, A\&SA 422, L33

Muecket, J.P., Petitjean, P., Kates, R.E. \& Riediger, R. 1996, A\&\&A 308, 17

Murdoch, H.S., Hunstead, R.W., Pettini, M. \& Blades, J.C. 1986, ApJ 309, 19

Murphy, M.T., Webb, J., Flambaum, V., Prochaska, J.X. \& Wolfe, A.M. 2001, MNRAS 327, 1237

Murphy, M.T., Webb, J.K. \& Flambaum, V.V. 2003, MNRAS 345, 609

Petitjean, P., Srianand, R. \& Ledoux, C. 2000, A\& A 364, L26

Petitjean, P., Srianand, R. \& Ledoux, C. 2002, MNRAS 332, 383

Pettini, M., Smith, L.J., King, D.L. \& Hunstead, R.W. 1997, ApJ 486, 665

Prochaska, J. \& Wolfe, A.M. 1997, ApJ 487, 73

Prochaska, J. \& Wolfe, A.M. 2002, ApJ 566, 68

Prochaska, J., Gawiser, E., Wolfe, A.M., Castro, S. \& Djorgovski, S.G. 2003, ApJ 595, 9

Quast, R., Reimers, D. \& Levshakov, S.A. 2004, A\& $A$ 415, L7

Rao, S., Nestor, D.B., Turnshek, D.A., Lane, W.M., Monier, E.M. \& Bergeron, J. 2003, ApJ 595,94

Rauch, M., Miralda-Escude, J., Sargent, W,L.W., et al. 1997, ApJ 489, 7

Ricotti, M., Gnedin, N.Y. \& Shull, J.M. 2000, ApJ 534, 41

Rollinde, E., Srianand, R., Thuens, T., Petitjean, P. \& Chand, H. 2005, MNRAS 361, 1015

Savaglio, S., Glazebrook, K., Le Borgne, D. et al. 2005, ApJ 635, 260

Schaye, J., Theuns, T., Leonard, A. \& Efstathiou, G. 1999, MNRAS 310, 57

Schaye, J., Aguirre, A., Kim, T., Theuns, T., Rauch, M. \& Sargent, W.L.W. 2003, ApJ 596, 768

Schirber, M., Miralda-Escude, J. \& McDonald, P. 2004, ApJ, 610, 105

Scott, J., Bechtold, J., Dobrzycki, A. \& Kulkani, V.P. 2000, ApJS 130, 67

Seljak, U., Makarov, A., McDonald, P., et al. 2005, PhysRevD 71, 103515

Songaila, A. 2005, AJ 130, 1996

Songaila, A. \& Cowie, L. 1996, AJ 112, 335

Srianand, R., Petitjean, P. \& Ledoux, C. 2000, Nature 408, 931

Srianand, R., Chand, H., Petitjean, P. et al. 2004, PhysRevL 92, 121302

Srianand, R., Petitjean, P., Ledoux, C., Ferland, G. \& Shaw, G. 2005, MNRAS 362, 549

Srianand, R., Shaw, G., Ferland, G., Petitjean, P. \& Ledoux, C. 2005a, (astro-ph/0506556)

Theuns, T., Leonard, A., Efstathiou, G., Pearce, F.R. \& Thomas, P.A. 1998, MNRAS 301, 478

Tytler et al. 1995, in: Meylan, G., (ed.), QSO Absorption lines, Proc. ESO workshop Berlin, Springe-Verlag, Berlin, p. 289

Theuns, T., Schaye, J., Zaroubi, S. et al. 2002b, ApJ 567, L103

Weatherley, S.J., Warren, S.J., Moller, P., et al. 2005, MNRAS 358, 985

Wolfe, A.M. 1995, in: Meylan, G., (ed.), QSO Absorption lines, Proc. ESO workshop Berlin, Springer, p.13

Wolfe, A.M., Gawiser, E. \& Prochaska, J.X. 2003, ApJ 593, 235

Wolfe, A.M., Howk, J.C., Gawiser, E., Prochaska, J.X. \& Lopez, S. 2004, ApJ 615, 625

Zaldarriaga, M., Hui, L. \& Tegmark, M. 2001, ApJ 557, 519

Zhang, Y., Anninos, P., Norman, M. \& Meiksin, A. 1997, ApJ 485, 496 